\title{
A Global Regularity Result for the 2D Generalized Magneto-Micropolar Equations
}

\author{
Hui Zhang (iD \\ College of Mathematical and Physical Science, Anqing Normal University, Anqing 246133, China \\ Correspondence should be addressed to Hui Zhang; zhangaqtc@126.com
}

Received 30 December 2021; Accepted 28 January 2022; Published 24 February 2022

Academic Editor: Salah Mahmoud Boulaaras

Copyright $\odot 2022$ Hui Zhang. This is an open access article distributed under the Creative Commons Attribution License, which permits unrestricted use, distribution, and reproduction in any medium, provided the original work is properly cited.

In this paper, we proved the global (in time) regularity for smooth solution to the 2D generalized magneto-micropolar equations with zero viscosity. When there is no kinematic viscosity in the momentum equation, it is difficult to examine the bounds on the any derivatives of the velocity $\left\|J^{\varepsilon} u\right\|_{L^{2}}$. In order to overcome the main obstacle, we find a new unknown quantity which is by combining the vorticity and the microrotation angular velocity; the structure of the system including the combined quantity obeys a Beale-Kato-Majda criterion. Moreover, the maximal regularity of parabolic equations together with the classic commutator estimates allows us to derive the $H^{s}$ estimates for solutions of the system.

\section{Introduction}

The standard 3D incompressible magneto-micropolar equations can be written as

$$
\left\{\begin{array}{l}
\partial_{t} u+u \cdot \nabla u-(\mu+\kappa) \Delta u+\nabla \pi=b \cdot \nabla b+2 \kappa \nabla \times \omega, \\
\partial_{t} \omega+u \cdot \nabla \omega-\gamma \Delta \omega-\nu \nabla \nabla \cdot \omega+4 \kappa \omega=2 \kappa \nabla \times u, \\
\partial_{t} b+u \cdot \nabla b-\eta \Delta b=b \cdot \nabla u, \\
\nabla \cdot u=\nabla \cdot b=0, \\
(u, \omega, b)(x, 0)=\left(u_{0}(x), \omega_{0}(x), b_{0}(x)\right) .
\end{array}\right.
$$

where $\quad u(x, t) \in \mathbb{R}^{3}, \omega(x, t) \in \mathbb{R}^{3}, b(x, t) \in \mathbb{R}^{3}, \quad$ and $\pi=\pi(x, t) \in \mathbb{R}$ denote the velocity of the fluidthe microrotational velocity, magnetic field, and pressure, respectively. $\mu$ is the kinematic viscosity, $\kappa$ is the vortex viscosity, $\gamma$ and $\nu$ are spin viscosities, and $\eta$ is the magnetic diffusivity. $\left(u_{0}, \omega_{0}, b_{0}\right)$ are the given initial data with $\nabla \cdot u_{0}=\nabla \cdot b_{0}=0$.

The magneto-micropolar equations have been extensively studied and applied by many engineers and physicists [1]. The mathematical study stared in the 1970s and has attracted considerable attentions. Rojas-Medar and Boldrin
[2] proved the existence of weak solutions by the Galerkin method and, in 2D case, also proved the uniqueness of the weak solutions. Ortega-Torres and Rojas-Medar [3] established the local in time existence and uniqueness of strong solutions and proved global in time existence of strong solution for small initial data. However, whether the local strong solutions can exist globally or the global weak solution is regular and unique is an outstanding open problem. There are many interesting results about the theory of global well-posedness of solution to the Cauchy problem and initial-boundary-value problem for system (1) in [4-14] and the references therein.

The 3D magneto-micropolar equations reduce 2D magneto-micropolar equations when

$$
\begin{array}{ll}
u=\left(u_{1}\left(x_{1}, x_{2}, t\right), u_{2}\left(x_{1}, x_{2}, t\right), 0\right), & \pi=\pi\left(x_{1}, x_{2}, t\right), \\
b=\left(b_{1}\left(x_{1}, x_{2}, t\right), b_{2}\left(x_{1}, x_{2}, t\right), 0\right), & \omega=\left(0,0, w_{3}\left(x_{1}, x_{2}, t\right)\right) .
\end{array}
$$

More explicitly, the 2D magneto-micropolar equations can be written as 


$$
\left\{\begin{array}{l}
\partial_{t} u+u \cdot \nabla u-(\mu+\kappa) \Delta u+\nabla \pi=b \cdot \nabla b+2 \kappa \nabla \times \omega \\
\partial_{t} \omega+u \cdot \nabla \omega-\gamma \Delta \omega+4 \kappa \omega=2 \kappa \nabla \times u \\
\partial_{t} b+u \cdot \nabla b-\eta \Delta b=b \cdot \nabla u \\
\nabla \cdot u=\nabla \cdot b=0 \\
(u, \omega, b)(x, 0)=\left(u_{0}(x), \omega_{0}(x), b_{0}(x)\right)
\end{array}\right.
$$

where we have written $u=\left(u_{1}, u_{2}\right)$ and $\omega$ is for $\omega_{3}$ for notational brevity. It is worth noting that, in the $2 \mathrm{D}$ case,

$$
\Omega=\nabla \times u=\partial_{1} u_{2}-\partial_{2} u_{1}
$$

is a scalar function and $\nabla \times \omega=\left(\partial_{2} w,-\partial_{1} \omega\right)$ is a vector function.

The well-posedness problem about 2D magnetomicropolar equations and closely related equations such as the micropolar equations $(b=0)$ has attracted considerable attention, for example, [15-18]. In [17], Dong et al. studied micropolar equations with partial dissipation case which involves no velocity dissipation. They introduced a new known quantity to obtain the existence and uniqueness of classical solutions.

This paper aims at the fractional dissipation case, when (3) involves no velocity dissipation. More precisely, we study the existence and uniqueness of classical solutions to the $2 \mathrm{D}$ generalized magneto-micropolar equations:

$$
\left\{\begin{array}{l}
\partial_{t} u+\kappa u+\nabla \pi+u \cdot \nabla u=b \cdot \nabla b+2 \kappa \nabla \times \omega, \\
\partial_{t} \omega+u \cdot \nabla \omega-\gamma \Delta \omega+4 \kappa \omega=2 \kappa \nabla \times u, \\
\partial_{t} b+u \cdot \nabla b+(-\Delta)^{\beta} b=b \cdot \nabla u, \\
\nabla \cdot u=\nabla \cdot b=0, \\
(u, \omega, b)(x, 0)=\left(u_{0}(x), \omega_{0}(x), b_{0}(x)\right) .
\end{array}\right.
$$

We remark that the term $\kappa u$ does not play any significant role in the global regularity problem. It is kept in (5) simply to reflect the fact that the magneto-micropolar equations require the presence of the microrotational effect and microrotational inertia, namely, $\kappa>0$. We establish the following global regularity result for (5).

Theorem 1. Let $1<\beta<2$ and $\left(u_{0}, \omega_{0}, b_{0}\right) \in H^{s}\left(\mathbb{R}^{2}\right)(s>2)$ with $\nabla \cdot u_{0}=\nabla \cdot b_{0}=0$. Then, the $2 D$ magneto-micropolar equation (5) has a unique global solution $(u, \omega, b)$ satisfying, for $\forall t>0$,

$$
u, \omega, b \in L^{\infty}\left([0, t) ; H^{s}\left(\mathbb{R}^{2}\right)\right) ; \omega \in L^{2}\left([0, t) ; H^{s+1}\left(\mathbb{R}^{2}\right)\right) ; \quad b \in L^{2}\left([0, t) ; H^{s+\beta}\left(\mathbb{R}^{2}\right)\right)
$$

Remark 1. It should be mentioned that, in [19], Fan and Zhao proved a similar result to MHD equations (when $\kappa=0$ and $\omega=0)$. However, due to the presence of microrotational term, the corresponding method is difficult to be used directly. To overcome this difficulty, we will introduce a new unknown term to obtain the desired result.

Throughout this paper, we use $L^{p}\left(\mathbb{R}^{2}\right)$ to denote the standard Lebesgue and Sobolev spaces, respectively. We use $H^{m}\left(\mathbb{R}^{2}\right)$ to denote the $\|f\|_{L^{p}}$ norm of $L^{p}\left(\mathbb{R}^{2}\right)$. The letter $f$ denotes various positive and finite constants.

\section{Proof of Theorem 1}

This section is devoted to the Proof of Theorem 1. The existence and uniqueness of local smooth solutions can be done by a classical method on some fixed time interval $C$, see, for example, [20]. Thus, it is $[0, T)$ enough to established a priori estimates for any given $t>0$.

Proposition 1. Assume $\left(u_{0}, \omega_{0}, b_{0}\right)$ obeys the assumptions stated in Theorem 1 with $1<\beta<2$. Let $(u, \omega, b)$ be the corresponding solution; then, we have the global $L^{2}$ bounds of

$\|(u, \omega, b)\|_{L^{2}}^{2}+\gamma \int_{0}^{t}\|\nabla \omega(\tau)\|_{L^{2}}^{2} \mathrm{~d} \tau+\int_{0}^{t}\left\|J^{\beta} b(\tau)\right\|_{L^{2}}^{2} \mathrm{~d} \tau \leq C$,

for any $t>0$, which implies $\int_{0}^{t}\|b(\tau)\|_{L^{\infty}}^{2} d \tau<+\infty$ due to $\beta>1$.

Proof of Proposition 1. Taking $L^{2}$ inner product of equations (5) and integrating by parts and using $\nabla \cdot u=\nabla \cdot b=0$, we obtain

$$
\frac{1}{2} \frac{d}{d t}\|(u, \omega, b)\|_{L^{2}}^{2}(\tau)+\kappa\|u(\tau)\|_{L^{2}}^{2}+4 \kappa\|\omega(\tau)\|_{L^{2}}^{2}+\gamma\|\nabla \omega(\tau)\|_{L^{2}}^{2}+\left\|J^{\beta} b(\tau)\right\|_{L^{2}}^{2}=0
$$

where we have used the following fact:

$$
\begin{aligned}
& \int_{\mathbb{R}^{2}} b \cdot \nabla b \cdot u \mathrm{~d} x+\int_{\mathbb{R}^{2}} b \cdot \nabla u \cdot b \mathrm{~d} x=0, \\
& \int_{\mathbb{R}^{2}} \nabla \times \omega \cdot u \mathrm{~d} x+\int_{\mathbb{R}^{2}} \nabla \times u \cdot \omega=0 .
\end{aligned}
$$

Integrating (8) in time from 0 to $t$ implies (7).

Now, we give the global $H^{1}$ bound for $u, \omega$, and $b$.

Proposition 2. Under the same condition on Proposition 1, then we have 
$\|(\Omega, \nabla \omega, j)\|_{L^{2}}^{2}(t)+\gamma \int_{0}^{t}\|\Delta \omega(\tau)\|_{L^{2}}^{2} \mathrm{~d} \tau+\int_{0}^{t}\left\|J^{\beta} j(\tau)\right\|_{L^{2}}^{2} \mathrm{~d} \tau \leq C$,

for any $t>0$.

Proof of Proposition 2. There we denote $\Omega=\nabla \times u$ and $j=\nabla \times b$; then, from the u-equation of (5), we have

$$
\partial_{t} \Omega+\kappa \Omega+u \cdot \nabla \Omega=b \cdot \nabla j-2 \kappa \Delta \omega .
$$

Taking the $L^{2}$ inner product to equation (9) with $\Omega$ and integrating by parts yield

$$
\frac{1}{2} \frac{d}{d t}\|\Omega\|_{L^{2}}^{2}+\kappa\|\Omega\|_{L^{2}}^{2}=\int_{\mathbb{R}^{2}} b \cdot \nabla j \cdot \Omega \mathrm{d} x-2 \kappa \int_{\mathbb{R}^{2}} \Delta \omega \cdot \Omega d x=I_{1}+I_{2} .
$$

Then, from the b-equation of (5), we have

$$
Q(\nabla u, \nabla b)=2 \partial_{1} b_{1}\left(\partial_{1} u_{2}+\partial_{2} u_{1}\right)+2 \partial_{2} u_{2}\left(\partial_{1} b_{2}+\partial_{2} b_{1}\right)
$$

$$
\partial_{t} j+u \cdot \nabla j+(-\Delta)^{\beta} j=b \cdot \nabla \Omega+Q(\nabla u, \nabla b),
$$

with

Taking the $L^{2}$ inner product to the equation (11) with $j$ and integrating by parts yield

$$
\frac{1}{2} \frac{d}{\mathrm{~d} t}\|j\|_{L^{2}}^{2}+\left\|J^{\beta} j\right\|_{L^{2}}^{2}=\int_{\mathbb{R}^{2}} b \cdot \nabla \Omega \cdot j d x+\int_{\mathbb{R}^{2}} Q(\nabla u, \nabla b) \cdot j \mathrm{~d} x=I_{3}+I_{4}
$$

Taking the $L^{2}$ inner product to the $\omega$-equation to (5) with $-\Delta \omega$ and integrating by parts yield

$$
\frac{1}{2} \frac{d}{d t}\|\nabla \omega\|_{L^{2}}^{2}+\gamma\|\Delta \omega\|_{L^{2}}^{2}+4 \kappa\|\nabla \omega\|_{L^{2}}^{2}=\int_{\mathbb{R}^{2}} u \cdot \nabla \omega \cdot \Delta \omega \mathrm{d} x-2 \kappa \int_{\mathbb{R}^{2}} \Omega \cdot \Delta \omega \mathrm{d} x=I_{5}+I_{6}
$$

Because of the divergence-free conditions, we have

$$
\int_{\mathbb{R}^{2}} b \cdot \nabla j \cdot \Omega \mathrm{d} x+\int_{\mathbb{R}^{2}} b \cdot \nabla \Omega \cdot j \mathrm{~d} x=0 .
$$

We now estimate $I_{2}, I_{4}$ and $I_{5}, I_{6}$.

Through integrating by parts and applying Holder's inequality, Young inequality, and Sobolev's inequalities, we have

$$
\begin{aligned}
\left|I_{2}\right|,\left|I_{6}\right| & \leq\|\Omega\|_{L^{2}}\|\Delta \omega\|_{L^{2}} \leq C\|\Omega\|_{L^{2}}^{2}+\frac{\gamma}{4}\|\Delta \omega\|_{L^{2}}^{2}, \\
\left|I_{4}\right| & \leq \int_{\mathbb{R}^{2}}|\nabla u \| \nabla b|^{2} \mathrm{~d} x \\
& \leq C\|\Omega\|_{L^{2}}\|j\|_{L^{2}}^{2} \\
& \leq C\|\Omega\|_{2}\|b\|_{L^{\infty}}^{2 \beta-1 / \beta}\left\|J^{\beta} j\right\|_{L^{2}}^{1 / \beta} \\
& \leq C\|\Omega\|_{2}^{2 \beta / 2 \beta-1}\|b\|_{L^{\infty}}^{2}+\frac{1}{2}\left\|J^{\beta} j\right\|_{L^{2}}^{2} \\
& \leq C\|\Omega\|_{2}^{2}\|b\|_{L^{\infty}}^{2}+C\|b\|_{L^{\infty}}^{2}+\frac{1}{2}\left\|J^{\beta} j\right\|_{L^{2}}^{2}, \\
\left|I_{5}\right| & \leq \int_{\mathbb{R}^{2}}|\nabla u \| \nabla \omega|^{2} \mathrm{~d} x \\
& \leq C\|\Omega\|_{L^{2}}\|\nabla \omega\|_{L^{4}}^{2} \\
& \leq C\|\Omega\|_{L^{2}}^{2}\|\nabla \omega\|_{L^{2}}^{2}+\frac{\gamma}{4}\|\nabla \omega\|_{L^{2}}^{2},
\end{aligned}
$$

Then, we have the following inequality:

$$
\begin{aligned}
& \frac{1}{2} \frac{d}{d t}\|(\Omega, \nabla \omega, j)\|_{L^{2}}^{2}+\kappa\|\Omega\|_{L^{2}}^{2}+4 \kappa\|\nabla \omega\|_{L^{2}}^{2} \\
& +\frac{1}{2}\left\|J^{\beta} j\right\|_{L^{2}}^{2}+\frac{\gamma}{2}\|\Delta \omega\|_{L^{2}}^{2} \\
& \leq C\|\Omega\|_{L^{2}}^{2}\left(\|\nabla \omega\|_{L^{2}}^{2}+\|b\|_{L^{\infty}}^{2}\right)+C\|b\|_{L^{\infty}}^{2} .
\end{aligned}
$$

Integrating (19) in time from 0 to $t$ and combining with Proposition 1 implies (20).

Proposition 3. Under the same condition on Proposition 1, then we have

$$
\left\|J^{\beta} b\right\|_{L^{2}}^{2}+\int_{0}^{t}\left\|J^{2 \beta} b\right\|_{L^{2}}^{2} \mathrm{~d} \tau \leq C
$$

for any $t>0$.

Proof of Proposition 3. Testing $b$ - equation of (5) with $(-\Delta)^{\beta} b$, we have 


$$
\begin{aligned}
& \frac{1}{2} \frac{d}{\mathrm{~d} t}\left\|J^{\beta} b\right\|_{L^{2}}^{2}+\left\|J^{2 \beta} b\right\|_{L^{2}}^{2}=\int(b \cdot \nabla u-u \cdot \nabla b) J^{2 \beta} b \mathrm{~d} x \\
& \leq\left(\|b\|_{L^{\infty}}\|\nabla u\|_{L^{2}}+\|u\|_{L^{4}}\|\nabla b\|_{L^{4}}\right)\left\|J^{2 \beta} b\right\|_{L^{2}} \\
& =I_{1}+I_{2} .
\end{aligned}
$$

We now estimate $I_{1}$, due to Proposition 2,

$$
\left|I_{1}\right| \leq C\|b\|_{L^{\infty}}^{2}+\frac{1}{4}\left\|J^{2 \beta} b\right\|_{L^{2}}^{2} .
$$
have

Applying Sobolev's inequality and Young inequality, we

$$
\left|I_{2}\right| \leq C\left\|J^{2 \beta} b\right\|_{L^{2}}^{1+(3 / 4 \beta)} \leq C+\frac{1}{4}\left\|J^{2 \beta} b\right\|_{L^{2}}^{2} .
$$

Collecting the estimates of $I_{1}$ and $I_{2}$, we have

$$
\frac{d}{\mathrm{~d} t}\left\|J^{\beta} b\right\|_{L^{2}}^{2}+\left\|J^{2 \beta} b\right\|_{L^{2}}^{2} \leq C+C\|b\|_{L^{\infty}}^{2}
$$

Integrating (24) in time from 0 to $t$ implies (20).

Due to $\beta>1,(20)$ implies, for any $p \in(2,+\infty]$, $b \in L^{\infty}\left(0, t ; L^{\infty}\left(\mathbb{R}^{2}\right)\right) ; \int_{0}^{t}\|\nabla b(\tau)\|_{L^{q}}^{2} \mathrm{~d} \tau \leq C$, for $\forall t>0$.

Proposition 4. Under the same condition on Proposition 1, then corresponding solutions $(u, \omega, b)$ admits the following global bounds, for any $q \in(2,+\infty)$ and any $t>0$, we have

$$
\Delta \omega ;(-\Delta)^{\beta} b \in L^{2}\left(0, t ; L^{q}\left(\mathbb{R}^{2}\right)\right) ; \Omega \in L^{\infty}\left(0, t ; L^{q}\left(\mathbb{R}^{2}\right)\right),
$$

for any $t>0$.

We first recall the maximal regularity of parabolic equation $[19,21]$.

Lemma 1. For the parabolic equation,

$$
\partial_{t} u+(-\Delta)^{\beta} u=f
$$

with the initial data $u(x, 0)=u_{0}(x)$. Let $\beta \geq 1,2<q<\infty$, we have

$$
\int_{0}^{t}\left\|J^{2 \beta} u\right\|_{L^{q}}^{2} \mathrm{~d} \tau \leq C\left(u_{0}\right)+\int_{0}^{t}\|f\|_{L^{q}}^{2} \mathrm{~d} \tau
$$

for any $t>0$.

From the $b$ - equation of (5), we have

$$
\partial_{t} b+(-\Delta)^{\beta} b=b \cdot \nabla u-u \cdot \nabla b .
$$

Due to $W^{1, p}\left(\mathbb{R}^{2}\right) \longrightarrow L^{\infty}\left(\mathbb{R}^{2}\right),(p>2)$, so we have

$$
\|b \cdot \nabla u-u \cdot \nabla b\|_{L^{q}} \leq\left(\|b\|_{L^{\infty}}+\|\nabla b\|_{q}\right)\|\nabla u\|_{L^{q}} .
$$

Then, from Lemma 1, we have

$$
\int_{0}^{t}\left\|\left(-\Delta^{\beta}\right) b(\tau)\right\|_{L^{q}}^{2} \mathrm{~d} \tau \leq C \int_{0}^{t}\left(\|b(\tau)\|_{L^{\infty}}^{2}+\|\nabla b(\tau)\|_{q}^{2}\right)\|\nabla u(\tau)\|_{L^{q}}^{2} \mathrm{~d} \tau .
$$

From the $\omega$-equation of (5), we have

$$
\partial_{t} \omega-\gamma \Delta \omega=2 \kappa \Omega-4 \kappa \omega-u \cdot \nabla \omega .
$$

Then, from Lemma 1, we have

$$
\int_{0}^{t}\|\Delta \omega(\tau)\|_{p}^{2} \mathrm{~d} \tau \leq C \int_{0}^{t}\|\Delta \omega(\tau)\|_{p}^{2}\|\nabla u(\tau)\|\left\|_{L^{p}}^{2} d \tau+\int_{0}^{t}\right\| \Omega(\tau) \|_{p}^{2} \mathrm{~d} \tau .
$$

Proof of Proposition 4. Testing (10), by $|\Omega|^{p-2} p(2<p<\infty)$, we obtain

$\frac{1}{p} \frac{d}{d t}\|\Omega\|_{L^{p}}^{p} \leq C\|b\|_{L^{\infty}}\|\nabla j\|_{L^{p}}\|\Omega\|_{L^{p}}^{p-1}+C\|\Delta \omega\|_{L^{p}}\|\Omega\|_{L^{p}}^{p-1}$

Then, we have

$$
\frac{d}{d t}\|\Omega\|_{L^{p}}^{2} \leq C\left(\|\nabla j\|_{L^{p}}^{2}+\|\Delta \omega\|_{L^{p}}^{2}\right)+C\|\Omega\|_{L^{p}}^{2}
$$

Thus, integrating the above inequality in time, we derive

$$
\begin{aligned}
\|\Omega\|_{L^{p}}^{2} & \leq C+\int_{0}^{t}\left(\|\nabla j\|_{L^{p}}^{2}+\|\Delta \omega\|_{L^{p}}^{2}+C\|\Omega\|_{L^{p}}^{2}\right) \mathrm{d} \tau \\
& \leq C+C \int_{0}^{T}\left((-\Delta)^{\beta}\|b\|_{L^{p}}^{2}+\|\Delta \omega\|_{L^{p}}^{2}+C\|\Omega\|_{L^{p}}^{2}\right) \mathrm{d} \tau \\
& \leq C+C \int_{0}^{T}\left(\|b\|_{L^{\infty}}^{2}+\|\nabla b\|_{L^{p}}^{2}+\|\nabla \omega\|_{L^{p}}^{2}\right)\|\Omega\|_{L^{p}}^{2} \mathrm{~d} \tau .
\end{aligned}
$$

Applying the Gronwall inequality, we have

$$
\|\Omega\|_{L^{\infty}\left(0, t ; L^{p}\right)} \leq C \text {. }
$$

Furthermore, combining (31), (33), and (37), we have

$$
\int_{0}^{t}\left\|(-\Delta)^{\beta} b(\tau)\right\|_{q}^{2} \mathrm{~d} x \leq C ; \int_{0}^{T}\|\Delta \omega(\tau)\|_{p}^{2} \mathrm{~d} \tau \leq C .
$$

Proposition 5. Under the same condition on Proposition 1, then corresponding solutions $(u, \omega, b)$ admits the following global bounds, for any $t>0$,

$$
\Omega \in L^{1}\left(0, t ; L^{\infty}\left(\mathbb{R}^{2}\right)\right) .
$$

Proof of Proposition 5. We set $Z=\Omega+2 \kappa / \gamma \omega$; then, we have

$$
\partial_{t} Z+u \cdot \nabla Z+\left(\kappa-\frac{4 \kappa^{2}}{\gamma}\right) Z+\left(\frac{8 \kappa^{3}}{\gamma^{2}}+\frac{6 \kappa^{2}}{\gamma}\right) \omega=b \cdot \nabla j
$$

Multiplying (40) by $|Z|^{p-2} Z$ with $2<p<\infty$ and integrating in $\mathbb{R}^{2}$, we have 


$$
\begin{aligned}
\frac{1}{p} \frac{d}{d t}\|Z(t)\|_{L^{p}}^{p} & \leq\left|\int_{\mathbb{R}^{2}}\left[\left(\kappa-\frac{4 \kappa^{2}}{\gamma}\right) Z+\left(\frac{8 \kappa^{3}}{\gamma^{2}}+\frac{6 \kappa^{2}}{\gamma}\right) \omega+b \cdot \nabla j\right]\right|\left|Z^{p-2} Z \mathrm{~d} x\right| \\
& \leq C\|Z\|_{L^{p}}^{p}+C\left(\|\omega\|_{L^{p}}+\|b \cdot \nabla j\|_{L^{p}}\right)\|Z\|_{L^{p}}^{p-1} .
\end{aligned}
$$

Applying Gronwall inequality, one has

$$
\|Z(t)\|_{L^{p}} \leq C\left(\|Z(0)\|_{L^{p}}+C \int_{0}^{T}\|\omega(\tau)\|_{L^{p}}+\|b \cdot \nabla j\|_{L^{p}}\right) \mathrm{d} \tau,
$$

We obtain, by letting $p \longrightarrow+\infty$,

$\|Z(t)\|_{L^{\infty}} \leq C\|Z(0)\|_{L^{\infty}}+C \int_{0}^{t}\|\omega(\tau)\|_{L^{\infty}}+\|b \cdot \nabla j\|_{L^{\infty}} \mathrm{d} \tau \leq C$,

and

$$
\|\Omega(t)\|_{L^{\infty}} \leq\|Z(t)\|_{L^{\infty}}+\|\omega(t)\|_{L^{\infty}} .
$$

Integrating (44) in time from 0 to $t$ implies (39).
Due to above estimates, we can prove (1). First, we recall commutator estimates [22] in the following. Let $J=(I-\Delta)^{1 / 2}$ denote the inhomogeneous differentiation operator; the proof of this lemma is based on some results due to Coifman and Meyer [23].

Lemma 2. Let $s>0$. Let $p, p_{1}, p_{3} \in(1, \infty)$ and $p_{2}, p_{4} \in[1, \infty]$ satisfy

$$
\frac{1}{p}=\frac{1}{p_{1}}+\frac{1}{p_{2}}=\frac{1}{p_{3}}+\frac{1}{p_{4}} .
$$

Then, for two constants $C_{1}$ and $C_{2}$,

$$
\begin{gathered}
\left\|J^{s}(f g)\right\|_{L^{p}} \leq C_{1}\left(\left\|J^{s} f\right\|_{L^{p_{1}}}\|g\|_{L^{p_{2}}}+\left\|J^{s} g\right\|_{L^{p_{3}}}\|f\|_{L^{p_{4}}}\right), \\
\left\|J^{s}(f g)-f J^{s} g\right\|_{L^{p}} \leq C_{2}\left(\left\|J^{s} f\right\|_{L^{p_{1}}}\|g\|_{L^{p_{2}}}+\left\|J^{s-1} g\right\|_{L^{p_{3}}}\|\nabla f\|_{L^{p_{4}}}\right) .
\end{gathered}
$$

Proof of Theorem 1. Applying $J^{s}$ with $s>2$ to system (5), taking the $L^{2}$ inner product with $J^{s} u, J^{s} \omega, J^{s} b$, respectively, and then adding them up, we can obtain

$$
\begin{aligned}
& \frac{1}{2} \frac{d}{d t}\left\|\left(J^{s} u, J^{s} \omega, J^{s} b\right)\right\|_{L^{2}}^{2}+\kappa\left\|J^{s} u\right\|_{L^{2}}^{2}+4 \kappa\left\|J^{s} \omega\right\|_{L^{2}}^{2}+\gamma\left\|J^{s} \Lambda \omega\right\|_{L^{2}}^{2}+\left\|J^{s} \Lambda^{\beta} b\right\|_{L^{2}}^{2} \\
& =2 \kappa \int J^{s}(\nabla \times \omega) J^{s} u+J^{s}(\nabla \times u) J^{s} \omega \mathrm{d} x-\int J^{s}(u \cdot \nabla u) J^{s} u \mathrm{~d} x+\int J^{s}(b \cdot \nabla b) J^{s} u \mathrm{~d} x \\
& \quad+\int J^{s}(u \cdot \nabla \omega) J^{s} \omega \mathrm{d} x-\int J^{s}(u \cdot \nabla b) J^{s} b+\int J^{s}(b \cdot \nabla u) J^{s} b=H_{1}+\cdots+H_{6} .
\end{aligned}
$$

Now, we estimate $H_{1}, H_{2}, H_{3}, H_{4}, H_{5}$, and $H_{6}$ one by one:

$$
\begin{aligned}
H_{1}= & 2 \kappa \int J^{s}(\nabla \times \omega) J^{s} u+J^{s}(\nabla \times u) J^{s} \omega \mathrm{d} x \\
& \leq \frac{8 \kappa^{2}}{\gamma}\left\|J^{s} u\right\|_{L^{2}}^{2}+\frac{\gamma}{2}\left\|J^{s} \Lambda \omega\right\|_{L^{2}}^{2}, \\
H_{2}= & \int\left[J^{s}, u \cdot \nabla u\right] \cdot J^{s} u \mathrm{~d} x \\
& \leq C\|\nabla u\|_{L^{\infty}}\left\|J^{s} u\right\|_{L^{2}}^{2} .
\end{aligned}
$$

Furthermore, we have 


$$
\left|H_{3}+H_{4}+H_{5}+H_{6}\right| \leq C\left(\|\nabla u\|_{L^{\infty}}+\|\nabla \omega\|_{L^{\infty}}+\|\nabla b\|_{L^{\infty}}\right)\left(\left\|\left(J^{s} u, J^{s} \omega, J^{s} b\right)\right\|_{L^{2}}^{2}\right) .
$$

Combining these estimates yield

$$
\begin{aligned}
& \frac{1}{2} \frac{d}{d t}\left\|\left(J^{s} u, J^{s} \omega, J^{s} b\right)\right\|_{L^{2}}^{2}+\kappa\left\|J^{s} u\right\|_{L^{2}}^{2}+4 \kappa\left\|J^{s} \omega\right\|_{L^{2}}^{2}+\frac{\gamma}{2}\left\|J^{s} \Lambda \omega\right\|_{L^{2}}^{2}+\left\|J^{s} \Lambda^{\beta} b\right\|_{L^{2}}^{2} \\
& \leq C\left(\|\nabla u\|_{L^{\infty}}+\|\nabla \omega\|_{L^{\infty}}+\|\nabla b\|_{L^{\infty}}\right)\left(\left\|\left(J^{s} u, J^{s} \omega, J^{s} b\right)\right\|_{L^{2}}^{2}\right)
\end{aligned}
$$

Combining with the above estimates and Gronwall's inequality, we can obtain the desired global $H^{s}$ bound.

\section{Data Availability}

All data used to support the findings of the study are included within the article.

\section{Conflicts of Interest}

The authors declare that they have no conflicts of interest.

\section{Acknowledgments}

H. Zhang was partially supported by NSFC (no. 31971185) and Anhui Education Bureau (no. KJ2019A0556).

\section{References}

[1] A. Eringen, "Theory of micropolar fluids," Indiana University Mathematics Journal, vol. 16, no. 1, pp. 1-18, 1966.

[2] M. A. Rojas-Medar and J. L. Boldrini, "Magneto-microploar fluid motion:existence of weak solutions," Internet.Rev. Mat. Comput.,vol. 11, pp. 443-460, 1998.

[3] M. A. Rojas-Medar, "Magneto-micropolar fluid motion: existence and uniqueness of strong solution," Mathematische Nachrichten, vol. 188, no. 1, pp. 301-319, 1997.

[4] Q. Chen and C. Miao, "Global well-posedness for the micropolar fluid system in critical Besov spaces," Journal of Differential Equations, vol. 252, no. 3, pp. 2698-2724, 2012.

[5] S. Gala, "On the improved regularity criterion of the solutions to the Navier-Stokes equations," Communications of the Korean Mathematical Society, vol. 35, pp. 339-345, 2020.

[6] K. Nakao and Y. Taniuchi, "An alternative proof of logarithmically improved Beale-Kato-Majda type extension criteria for smooth solutions to the Navier-Stokes equations," Nonlinear Analysis, vol. 176, pp. 48-55, 2018.

[7] M. A. Ragusa, "On weak solutions of ultraparabolic equations," Nonlinear Analysis: Theory, Methods \& Applications, vol. 47, no. 1, pp. 503-511, 2001.

[8] B.-Q. Dong and Z.-M. Chen, "Regularity criteria of weak solutions to the three-dimensional micropolar flows," Journal of Mathematical Physics, vol. 50, no. 10, p. 103525, 2009.

[9] B.-Q. Dong, Z. Chen, and Z.-M. Chen, "Asymptotic profiles of solutions to the $2 \mathrm{D}$ viscous incompressible micropolar fluid flows," Discrete \& Continuous Dynamical Systems-A, vol. 23, no. 3, pp. 765-784, 2009.
[10] S. Gala and R. Ma, "A regularity criterion for 3D micropolar fluid flows in terms of one partial derivative of the velocity," Annales Polonici Mathematici, vol. 116, pp. 217-228, 2016.

[11] E. Ortega-Torres and M. Rojas-Medar, "On the regularity for solutions of the micropolar fluid equations," Rendiconti del Seminario Matematico della Università di Padova, vol. 122, pp. 27-37, 2009.

[12] B. Yuan, "Magneto-microploar fluid motion: global existence of strong solutions," Acta Mathematica Scientia, vol. 30, pp. 1469-1480, 2010.

[13] H. Zhang and Y. Zhao, "Blow-up criterion for strong solution to the 3D magneto-micropolar fluid equations in the multiplier space," Acta Mathematica Scientia, vol. 188, pp. 1-7, 2012.

[14] Z. Zhang, Z.-a. Yao, and X. Wang, “A regularity criterion for the 3D magneto-micropolar fluid equations in Triebel-Lizorkin spaces," Nonlinear Analysis: Theory, Methods \& Applications, vol. 74, no. 6, pp. 2220-2225, 2011.

[15] R. Dipendra and J. Wu, "Global regularity for the 2D Magneto-Micropolar equations with partial dissipation," Journal of Mathematical Study, vol. 49, pp. 200-213, 2016.

[16] B. Dong and Z. Zhang, "Global regularity of the 2D micropolar fluid flows with zero angular viscosity," Journal of Differential Equations, vol. 249, pp. 169-194, 2010.

[17] B.-Q. Dong, J. Li, and J. Wu, "Global well-posedness and large-time decay for the 2D micropolar equations," Journal of Differential Equations, vol. 262, no. 6, pp. 3488-3523, 2017.

[18] K. Yamazaki, "Global regularity of the two-dimensional Magneto-Micropolar fluid system with zero angular viscosity," Discrete \& Continuous Dynamical Systems - A, vol. 35, no. 5, pp. 2193-2207, 2015.

[19] J. Fan and K. Zhao, "Global Cauchy problem of 2D generalized magnetohydrodynamic equations," Journal of Mathematical Analysis and Applications, vol. 420, no. 2, pp. 1024-1032, 2014.

[20] H. Bessaih, J. Chemin, and R. Danchin, "Fourier analysis and nonlinear partial differential equations," in Grundlehren der Mathematmatischen WissenschaftenSpringer, Berlin, Germany, 2011.

[21] H. Amann, "Maximal regularity for nonautonomous evolution equations," Advanced Nonlinear Studies, vol. 4, no. 4, pp. 417-430, 2004.

[22] T. Kato and G. Ponce, "Commutator estimates and the Euler and Navier-Stokes equations," Communications on Pure and Applied Mathematics, vol. 41, no. 7, pp. 891-907, 1988.

[23] R. R. Coifman and Y. Meyer, Au delá des opérateurs pseudodifférentieles, Asté,Mathématique de France, France, 1978. 